\title{
Simplicity of Amplitudes in Gravity and Yang-Mills Theories
}

\author{
N.E.J. Bjerrum-Bohr* \\ Niels Bohr International Academy, \\ Niels Bohr Institute, \\ Blegdamsvej 17, DK-2100, Copenhagen Ø, Denmark \\ E-mail: bjbohr@nbi.dk

\section{Pierre Vanhove} \\ Institut des Hautes Etudes Scientifiques, Le Bois-Marie, \\ F-91440 Bures-sur-Yvette, France \\ and \\ CEA, DSM, Institut de Physique Théorique, IPhT, CNRS, MPPU, \\ URA2306, Saclay, F-91191 Gif-sur-Yvette, France \\ E-mail: pierre.vanhove@cea.fr
}

\begin{abstract}
Computational techniques for amplitudes have, in recent years, seen rapid development. New results and techniques for amplitudes have significantly enhanced the calculational capabilities in gauge theories and gravity. Underlying the progress is a surprising simplicity of amplitude expressions. The origin of this simplicity is not yet understood, but it has the potential of leading to very interesting discoveries. Recently discussed has been the possibility of a twistor string theory formulation of Yang-Mills theory as well as the possibility of perturbative finiteness of $\mathscr{N}=8$ supergravity.
\end{abstract}

Workshop on Continuum and Lattice Approaches to Quantum Gravity September 17-192008

Brighton, University of Sussex, United Kingdom. ${ }^{\dagger}$

*Speaker.

†Preprint: IHES/P/09/26, IPHT-t09/077 


\section{Introduction}

The classical theory of General Relativity is very well tested at the scale of our solar system. This year, ninety years after the experimental verification of General Relativity by Eddington and Dyson on the island of Principe, the International Astronomy Year is being celebrated. Although the understanding and mastering of General Relativity effects beyond the Newtonian limit has lead to a high precision analysis of the energy loss of neutron star binary systems [1], the consequences of General Relativity as a quantum theory are still elusive both experimentally and theoretically. To understand the structure of our present universe the short comings of (quantum) gravity effects for cosmology will have to be understood.

Contrary to gauge theories of spin at most one, a theoretical analysis of the quantization of gravity is complicated by the non-linear nature of the gravitational interactions. It is possible to make an effective field theory description of gravity [2,3] however the complexity introduced by interactions and the consequences of diffeomorphism invariance, makes a traditional Feynman graph approach to perturbative gravity rather quickly intractable [4].

Witten's proposal [5] of gauge theory as a twistor string theory combined with powerful methods based on on-shell unitarity have led to a rapid new progress in computational techniques for scattering amplitudes. The past decade has seen crucial strides being taken in producing new results for various standard model processes in pure QCD, heavy quark physics, mixed QCD/electroweak processes (see [6] for a recent review) and on-shell gravity amplitudes in various dimensions. Surprising and crucial to the rapid progress have been a discovered simplicity of S-matrix elements in maximally supersymmetric gauge theories [7-9].

The application of on-shell unitarity methods greatly simplifies various computations by incorporating only physical gauge degrees-of-freedom. However symmetries are often not completely realized via such an approach and additional organizational principles are needed.

Clearly an optimal form for the tree level amplitude provide the first step and a theoretical guidance for higher loop computations. The simplicity of tree amplitudes explains in part simplicity at the loop level. But an additional understanding of the consequences of symmetries at loop orders is usually necessary for the full picture. In the case of theories without color factors the sum over all orderings of the external legs including all planar and non-planar contributions lead to important on-shell cancelations simplifying the structure of QED and gravity amplitudes [10-12]. The constraints from maximal supersymmetry is as well rather poorly understood in perturbation theory. For instance, in maximal supergravity four-graviton amplitudes display a much better ultraviolet behavior [4,13-18], than predicted by a superspace analysis [19-21].

We now know that loop amplitudes in $\mathscr{N}=4$ super-Yang-Mills which are leading in color enjoy a dual conformal symmetry and that one-loop amplitudes in $\mathscr{N}=8$ supergravity satisfy a no-triangle property $[10,11,22-28]$. Both of these properties put strong constraints on the basis of integral functions in which amplitudes can be expanded. These properties are consequences of the symmetries of the theory but are not so explicit in a unitarity based method. Their origin can be made more explicit by considering a string theory realization of these theories $[10,11,28]$.

In this talk we will discuss the recent progress for amplitudes in field theories without color factors. We will focus especially on results for $\mathscr{N}=8$ supergravity amplitudes. We will discuss new techniques for amplitude computations and consider the possibility of recycling progress for 
computations of Yang-Mills amplitudes into results for gravity amplitudes via the Kawai-LewellenTye relations. We will also discuss how it is possible to uncover a fascinating simplicity of gravity amplitudes via a combination of unitarity methods and the use of organizational inspiration from string theory.

\section{Amplitudes in Gauge Theory}

\subsection{Critical ultraviolet behavior of $\mathscr{N}=4$ perturbation}

The maximally supersymmetric case of $\mathscr{N}=4$ Yang-Mills is known to be perturbatively ultraviolet finite in $D \leq 4$ dimensions [20,29-31]. This can be shown by an implementation of half (eight) of the total (sixteen) supersymmetries in the theory. This implies that the $L$-loop four-gluon amplitude has a ultraviolet behavior dominated by

$$
\mathscr{A}_{4 ; L}^{(D)} \sim \Lambda^{(D-4) L-4} F^{4}
$$

where $\Lambda$ is a ultraviolet cutoff. This power counting law implies finiteness in dimensions $D \leq 4$. However, the situation is better than that because the CP-even term $F^{4}$ is related by supersymmetry to the CP-odd anomaly canceling term $B \wedge F^{4}$ where $B$ is the NS-NS B-field. One therefore expect these contributions to be one-loop exact [32-36]. This means that the $L>1$ contributions must have a low energy limit that behaves as $\partial^{2 \beta_{L}} F^{4}$ with $\beta_{L} \geq 1$ for $L \geq 1$, so that the prefactor contains at least two extra powers of momentum. In fact, direct perturbative evaluations of the four gluon amplitude $[37,38]$ indicates that $\beta_{L}=1$ for all $L>1$, leading to the ultraviolet behavior of ${ }^{1}$

$$
\mathscr{A}_{4 ; L}^{(D)} \sim \Lambda^{(D-4) L-6} \partial^{2} t_{8} \operatorname{tr}\left(F^{4}\right) \text { for } L \geq 2 .
$$

We refer to [40] for a discussion of the ultraviolet behavior of the sub-leading contributions in the color factors. In a unitarity computations the behavior of eq. (2.2) is directly obtained from an explicit realization of the supersymmetry, by considering diagrams [38] constructed from the 'rung rule' and via dual conformal symmetry $[9,41]$. The results from a unitarity based approach can be independently confirmed by superspace methods as in ref. [19-21].

At one-loop order in four dimensions one can expand all $n$-point amplitudes in a set of basis functions which consists of scalar boxes, triangles, bubble integrals and possibly rational polynomial functions $[42,43]$. This expansion in terms of basis functions is symbolically illustrated in figure 1. The split-up in basis functions holds via kinematic restrictions for four-dimensional momenta and because one-loop tensor integrals in amplitudes always can be reduced to scalar integrals $[42,44-46]$.

In $D=4-2 \varepsilon$ dimensions the one-loop $n$-point amplitude will take the schematic form

$$
\mathscr{A}_{n ; 1}^{(D)}=\mu^{2 \varepsilon} \int d^{D} \ell \frac{\mathscr{P}_{n}(\ell)}{\ell_{1}^{2} \cdots \ell_{n}^{2}}
$$

Here $\ell_{i}^{2}=\left(\ell-k_{1}-\cdots-k_{i}\right)^{2}$ are the propagators along the loop and $\mathscr{P}_{n}(\ell)$ is a polynomial in the loop momentum $\ell^{\mu}$. This polynomial is a function as well of the external momenta and the

\footnotetext{
${ }^{1}$ The tensor $t_{8}$ is defined in the appendix 9.A of volume II of [39].
} 


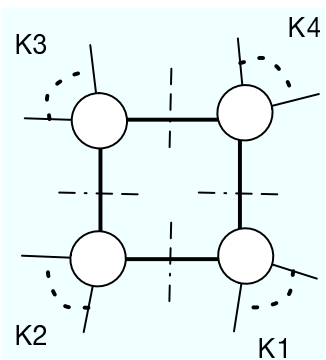

(a)

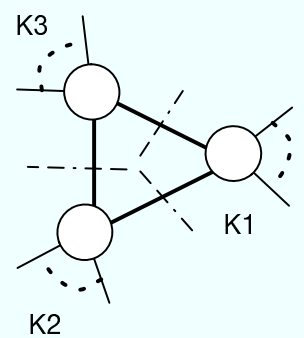

(b)



(c)

Figure 1: Basis of one-loop scalar integrals given by a) a scalar box with four propagators to be integrated over, b) scalar triangles with three propagators and c) a scalar bubble integral with only two propagators. In $D=4-2 \varepsilon$ dimensions these diagrams carry all the ultraviolet and infrared divergences of the amplitudes. Possible finite contributions to the amplitude are absorbed in a rational polynomial function.

helicities of the external states. The maximal dependence of the loop momentum $\ell$ is $n$ from the three point coupling of the Yang-Mills theory. From the saturation of half of the supersymmetries in the loop (i.e. eight supercharges) $n$ is reduced to $n-4$. Hence the numerator takes the form $\mathscr{P}_{n}(\ell)=\sum_{i} \mathscr{O}_{4}^{i} \times \mathscr{P}_{n-4}^{i}(\ell)$, where $\mathscr{O}_{4}^{i}$ is a dimension four operator made from external momenta and polarization vectors.

In each on-shell amplitude one can apply the following Passarino-Veltman reduction [47] which trade one power of loop momentum $\ell$ to a propagator

$$
\int d^{D} \ell \frac{2\left(\ell \cdot k_{1}\right)}{\ell^{2}\left(\ell-k_{1}\right)^{2}}(\cdots)=\int d^{D} \ell \frac{1}{\left(\ell-k_{1}\right)^{2}}(\cdots)-\int d^{D} \ell \frac{1}{\ell^{2}}(\cdots) .
$$

This identity is represented pictorially in figure 2 . Since the numerator in $n$-point $\mathscr{N}=4$ Super Yang-Mills one-loop amplitudes have at most $n-4$ powers of loop momenta after $n-4$ steps of reductions (2.4) the complete amplitude can decomposed completely in a basis of scalar box integral functions.
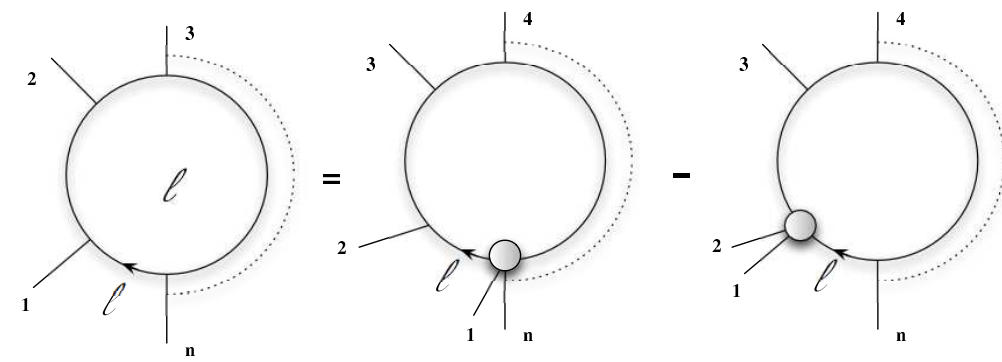

Figure 2: On-shell reduction of color ordered amplitudes.

\subsection{QED amplitudes}

We will now consider the one-loop n-photon amplitudes in massless QED. Furry's theorem apply here and dictates that amplitudes with an odd number of external photon lines are vanishing. Thus we only need to consider amplitudes with an even number of external lines. 
Each vertex will bring one power of momenta and make the numerator $\mathscr{P}_{n}(\ell)$ a polynomial with at most $n$ powers of loop momentum $\ell \equiv \ell_{n}$. Because of up to $n$ powers of loop momenta in the numerator of the $n$-point amplitude, one would expect via a succession of Passarino-Veltman reductions that the one-loop amplitude could be expanded in a linear basis of scalar box, triangle and bubble integral functions (see figure 1) and possible rational pieces.

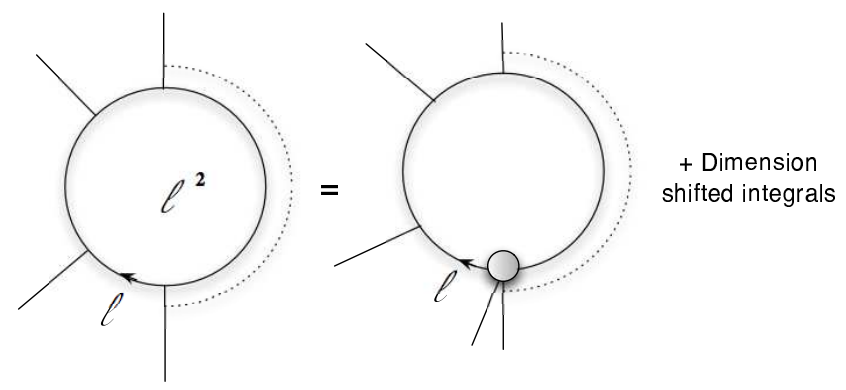

Figure 3: On-shell reduction of unordered amplitudes.

It was shown in ref. [12] that the one-loop $n$ photon amplitude in massless QED is completely expressible in terms of scalar box integral functions for $n \geq 8$ external photons. The cancelation of the scalar triangle and bubble functions was accounted for by the sum over the permutations imposed by the absence of color factors. This is a consequence of the fact that unordered colorless amplitudes satisfy a new type of reduction formula derived in [10] where two powers of loop momenta are traded for a propagator factor as depicted in figure 3. Such a reduction formula is reflecting the milder infrared behavior of colorless QED and gravity amplitudes compared to the QCD amplitudes [48].

The reduction formula in figure 3 shows the appearance of dimension shifted integrals. These can be shown to cancel in the physical amplitude by gauge invariance (In the string based formalism used in $[10,12]$ the cancelation makes use of a canceled propagator mechanism).

The application of this reduction formula gives that all one-loop amplitudes with $n \geq 8$ external photons can be expressed as a linear combination of scalar box integral functions

$$
\mathscr{A}_{n}^{1-\text { loop }}=\frac{i\left(\mu^{2}\right)^{\varepsilon}}{(4 \pi)^{2-\varepsilon}} \frac{\Gamma(1+\varepsilon) \Gamma^{2}(1-\varepsilon)}{\Gamma(1-2 \varepsilon)} \sum_{\alpha=\{1 m, 2 m e, 2 m h, 3 m, 4 m\}} \sum_{(i, j, k, l)} c^{\alpha}(i, j, k, l) F^{\alpha}(i, j, k, l) .
$$

Here $(i, j, k, l)$ is a parametrization of the four uncanceled propagators, defining the kinematic invariants of the scalar box functions [49]. We remark that this result is helicity independent and generalizes to all helicity configurations the result derived by Mahlon for MHV amplitudes in ref. [50].

We have in the previous equation introduced $F^{\alpha}$ which is the dimensionless scalar box function defined as in ref. [51]

$$
F^{\alpha}(i, j, k, l)=\frac{i(2 \pi)^{2-\varepsilon}}{2}\left(\left(x_{13}^{2} x_{24}^{2}-x_{12}^{2} x_{34}^{2}+x_{23}^{2} x_{41}^{2}\right)^{2}-4 x_{13}^{2} x_{23}^{2} x_{24}^{2} x_{41}^{2}\right)^{\frac{1}{2}} \int \frac{d^{4-2 \varepsilon} x_{0}}{(2 \pi)^{4-2 \varepsilon}} \prod_{i=1}^{4} \frac{1}{x_{0 i}^{2}},
$$

where $K_{i}^{\mu}=x_{i}^{\mu}-x_{i+1}^{\mu}$, with $1 \leq i \leq 4$ and $x_{5}=x_{1}$ and $K_{i}$ are the external momenta at each corner of the scalar box functions. We have used the notation $x_{i j}^{2}=\left(x_{i}^{\mu}-x_{j}^{\mu}\right)^{2}$. 
The sum is over the $d_{1}=n$ one-mass scalar box functions, the $d_{2 e}=n(n-5) / 2$ two-mass easy boxes, the $d_{2 h}=n(n-5)$ two-mass hard boxes, the $d_{3}=n(n-5)(n-6) / 2$ three-mass boxes, and the $d_{4}=n(n-5)(n-6)(n-7) / 24$ four-mass boxes [49]. Actually because of the soft limit relation between the two-mass easy and the one-mass scalar box functions we have,

$$
\lim _{K_{2} \rightarrow 0} F^{2 m e}\left(k_{i}, k_{j}+K_{1}, k_{k}, k_{l}+K_{2}\right)=F^{1 m}\left(k_{i}, k_{j}+K_{1}, k_{k}, k_{l}\right),
$$

thus there is no need to distinguish between these functions and separate them into two different sets. This gives a total of $n(n-3) / 2$ functions.

Because of the vanishing of the multi-photon tree level amplitudes no infrared singularities of the QED amplitudes are present

$$
\left.\mathscr{A}_{n}^{1-\text { loop }}\right|_{I R} \propto \sum_{i=1}^{n} \frac{\left(-\left(k_{i}+k_{i+1}\right)^{2}\right)^{-\varepsilon}}{\varepsilon} \mathscr{A}_{n}^{\text {tree }}=0 .
$$

This lead to $n(n-3) / 2$ relations for each independent kinematic invariant [49]. However the set of relations appear to be redundant. The vanishing of the infrared singularities imply that the one-loop multi-photon amplitudes are given by a ultraviolet and infrared finite combination of the scalar box functions. As well this guaranties that the amplitude is invariant under the dual conformal transformations of $[9,41]$.

\section{Amplitudes in Gravity}

The $D$-dimensional Einstein-Hilbert Lagrangian has the form:

$$
\mathscr{L}=\frac{1}{2 \kappa_{(4)}^{2}} \int d^{D} x \sqrt{-g} \mathscr{R},
$$

where $2 \kappa_{(4)}^{2}=2(2 \pi)^{2} G_{N}$. Ignoring renormalisation issues it is possible to make a formal quantization of this action. It is conventional to use $g_{\mu \nu} \equiv \eta_{\mu \nu}+\kappa_{(4)} h_{\mu \nu}$ and work in harmonic gauge: $\left(\partial^{\lambda} h_{\mu \lambda}=\frac{1}{2} \partial_{\mu} h_{\lambda}^{\lambda}\right)$. For this gauge choice the vertex rules for the three- and four-point Einstein vertices can be found to be $[52,53]$. However this traditional way of constructing amplitudes in gravity lead to very cumbersome and unmanageable results. Explicitly we have for instance

$$
\begin{aligned}
& V_{\mu \alpha, v \beta, \sigma \gamma}^{(3)}\left(k_{1}, k_{2}, k_{3}\right)= \\
& \frac{1}{2} \kappa_{(4)} \operatorname{sym}\left[-P_{3}\left(k_{1} \cdot k_{2} \eta_{\mu \alpha} \eta_{v \beta} \eta_{\sigma \gamma}\right)-P_{6}\left(k_{1 v} k_{1 \beta} \eta_{\mu \alpha} \eta_{\sigma \gamma}\right)+P_{3}\left(k_{1} \cdot k_{2} \eta_{\mu v} \eta_{\alpha \beta} \eta_{\sigma \gamma}\right)\right. \\
& +2 P_{6}\left(k_{1} \cdot k_{2} \eta_{\mu \alpha} \eta_{v \sigma} \eta_{\beta \gamma}\right)+4 P_{3}\left(k_{1 v} k_{1 \gamma} \eta_{\mu \alpha} \eta_{\beta \sigma}\right)-2 P_{3}\left(k_{1 \beta} k_{2 \mu} \eta_{\alpha \nu} \eta_{\sigma \gamma}\right)+2 P_{3}\left(k_{1 \sigma} k_{2 \gamma} \eta_{\mu v} \eta_{\alpha \beta}\right) \\
& \left.+2 P_{6}\left(k_{1 \sigma} k_{1 \gamma} \eta_{\mu \nu} \eta_{\alpha \beta}\right)+4 P_{6}\left(k_{1 v} k_{2 \gamma} \eta_{\beta \mu} \eta_{\alpha \sigma}\right)+4 P_{3}\left(k_{1 v} k_{2 \mu} \eta_{\beta \sigma} \eta_{\gamma \alpha}\right)-4 P_{3}\left(k_{1} \cdot k_{2} \eta_{\alpha \nu} \eta_{\beta \sigma} \eta_{\gamma \mu}\right)\right] .
\end{aligned}
$$

Here 'sym', denotes a symmetrization of each pair of indices: $(\mu \alpha),(v \beta), \ldots$ and the momentum factors: $\left(k_{1}, k_{2}, \ldots\right)$ are to be associated with the index pairs: $(\mu \alpha, v \beta, \ldots)$ correspondingly. The symbol: $\left(P_{\#}\right)$ means that a \#-permutation of indices and corresponding momenta has to 
carried out for this particular term. As seen the algebraic structure for the three-point vertex is already rather involved and complicated. At the level of Feynman diagram computations this vertex structure combined with the generic factorial increase in complexity with an increasing number of external legs makes it extremely difficult to compute amplitudes. Furthermore possible simplicity of expressions are hidden in very involved cancelations between a factorially increasing number of terms as the number of external legs increases.

In the on-shell amplitude the three-point vertex can be greatly simplified using the mass-shell relations $k_{i} \cdot k_{j}=0$ for $i, j=1,2,3$, and imposing the tracelessness condition $h_{1}^{\mu \mu}=0$

$V^{(3)}(1,2,3) \equiv h_{1}^{\mu \alpha} h_{2}^{v \beta} h_{3}^{\sigma \gamma} V_{\mu \alpha, v \beta, \sigma \gamma}^{(3)}\left(k_{1}, k_{2}, k_{3}\right)=\frac{\kappa_{(4)}}{2} h_{1}^{\mu \alpha} h_{2}^{v \beta} h_{3}^{\sigma \gamma}\left(k_{1 \mu} \eta_{v \sigma}+\right.$ perms $)\left(k_{1 \alpha} \eta_{\beta \gamma}+\right.$ perms $)$.

We see that the three points vertex in the on-shell amplitude actually takes the very suggestive form $V_{\text {gravity }}^{(3)} \sim\left(V_{Y M}^{(3)}\right)^{2}$ with $V_{Y M} \sim g_{Y M} h_{1}^{\mu} h_{2}^{v} h_{3}^{\rho}\left(k_{1 \mu} \eta_{v \sigma}+\right.$ perms $)$. This is a factorization which is highly surprising from the Lagrangian perspective (3.2). Such a relation indicates clearly that the structure of on-shell $n$-graviton amplitudes will take a simpler form than naïvely expected.

Field theory tree-level amplitudes can be derived in an infinite tension limit $\left(\alpha^{\prime} \rightarrow 0\right)$ of string graviton amplitudes. A general $n$-point scattering amplitude for a closed string will be connected to that of two open strings through [54]:

$$
\mathscr{M}_{n}^{\text {(closed string) }}=\kappa_{\sigma, \sigma^{\prime} \in S_{n} / \mathbb{Z}_{n}}^{n-2} e^{i \pi \Phi\left(\sigma, \sigma^{\prime}\right)} A_{n}^{\text {left (open string) }}(\sigma(1), \cdots, \sigma(n)) A_{n}^{\text {right (open string) }}\left(\sigma^{\prime}(1), \cdots, \sigma^{\prime}(n)\right) .
$$

Here $\sigma$ and $\sigma^{\prime}$ are permutations in $S_{n} / \mathbb{Z}_{n}$ of the set of all permutations, but with cyclic rotations removed and $\Phi\left(\sigma, \sigma^{\prime}\right)$ is a phase factor depending on the kinematics invariants. The open string amplitudes are the tree-level color-ordered gauge theory partial amplitudes $A_{n}^{\text {tree (open string) }}$ defined as

$$
\mathscr{A}_{n}^{\text {tree }}(1,2, \ldots, n)=g_{Y M}^{n-2} \sum_{\sigma \in S_{n} / Z_{n}} \operatorname{Tr}\left(T^{a_{\sigma(1)}} T^{a_{\sigma(2)}} \cdots T^{\left.a_{\sigma(n)}\right)}\right) A_{n}^{\text {tree }(\text { open string) }}(\sigma(1), \ldots, \sigma(n)) .
$$

The $T^{a_{i}}$ are fundamental representation matrices for the Yang-Mills gauge group $S U\left(N_{c}\right)$, normalized so that $\operatorname{Tr}\left(T^{a} T^{b}\right)=\delta^{a b}$.

In the field theory limit $\alpha^{\prime} \rightarrow 0$ this expression becomes

$$
\mathscr{M}_{n}^{\text {tree }}(1,2, \cdots, n)=\kappa^{n-2} \sum_{\sigma, \sigma^{\prime} \in S_{n} / \mathbb{Z}_{n}} f_{n-3}^{\sigma, \sigma^{\prime}}\left(s_{i j}\right) A_{n}^{\text {tree }}(\sigma(1), \cdots, \sigma(n)) \tilde{A}_{n}^{\text {tree }}\left(\sigma^{\prime}(1), \cdots, \sigma^{\prime}(n)\right),
$$

where $f_{n-3}^{\sigma, \sigma^{\prime}}\left(s_{i j}\right)$ is a homogeneous rational function of order $n-3$ in the kinematic invariants $s_{i j}=\left(k_{i}+k_{j}\right)^{2}$, such that $f_{n-3}^{\sigma, \sigma^{\prime}}\left(\lambda^{2} s_{i j}\right)=\lambda^{2(n-3)} f_{n-3}^{\sigma, \sigma^{\prime}}\left(s_{i j}\right)$, assuring that the gravity tree amplitude has the correct pole structures, and $A_{n}^{\text {tree }}(\sigma(1), \cdots, \sigma(n))$ is the field theory limit of the open string $n$-point amplitude.

The origin of the function $f_{n-3}^{\sigma, \sigma^{\prime}}\left(s_{i j}\right)$ can be understood as follows. If one rescales the external momenta as $k_{i} \rightarrow \lambda k_{i}$ the $n$-gluon Yang-Mills amplitude scale as

$$
A_{n}^{\text {tree }} \sim \lambda^{4-n}
$$


expressing the fact that the three- and four-point amplitudes lead to local interactions of respective form $\operatorname{tr}\left(\left[A_{\mu}, A_{v}\right] \partial^{[\mu} A^{v]}\right)$ and $\operatorname{tr}\left(\left[A_{\mu}, A_{v}\right]\left[A^{\mu}, A^{v}\right]\right)$. In the case of gravity since the Einstein-Hilbert action (3.1) gives contributions of order $\mathscr{O}\left(k^{2}\right)$ to any number of external graviton we must have,

$$
\mathscr{M}_{n}^{\text {tree }} \sim \lambda^{2}
$$

This implies that in the KLT relation (3.5) a rational function of order $n-3$ in the external momenta must multiply the product of Yang-Mills amplitudes. Tree-level amplitudes are presented in [54] in this form. The function $f^{\sigma, \sigma^{\prime}}\left(s_{i j}\right)$ is a polynomial of order $n-3$.

A particular case of the expression (3.5) is a left-right symmetric presentation of the gravity amplitude $[55,56]$ that is obtained by using the same basis of functions for the left and right moving open string amplitudes

$$
\mathscr{M}_{3}^{\text {tree }}(1,2,3)=-i \kappa\left|A_{3}^{\text {tree }}(1,2,3)\right|^{2}
$$

Using that

$$
A_{4}^{\text {tree }}(i, j, k, l)=\frac{1}{s_{i j} s_{k l}} t_{8}^{m_{1} \cdots m_{8}} \prod_{i=1}^{4} F_{m_{2 i-1} m_{2 i}}^{(i)}
$$

(with $F_{m n}^{(i)}=k_{m}^{i} h_{n}^{i}-k_{n}^{i} h_{m}^{i}$ and $(i, j, k, l)$ being a permutation of the labels of the external states $\{1,2,3,4\})$, it is immediate to massage the four-point KLT relation into a left/right-symmetric form

$$
\mathscr{M}_{4}^{\text {tree }}(1,2,3,4)=-i \kappa^{2}\left(s_{13}\left|A_{4}^{\text {tree }}(1,2,3,4)\right|^{2}+s_{12}\left|A_{4}^{\text {tree }}(1,3,2,4)\right|^{2}+s_{14}\left|A_{4}^{\text {tree }}(1,4,2,3)\right|^{2}\right) .
$$

At five-point order a left/right symmetric form valid for all helicity configurations has been presented in [56]

$$
\begin{aligned}
\mathscr{M}_{5}^{\text {tree }}(1,2,3,4,5) & =-i \kappa^{3}\left[c_{1}\left|A_{5}^{\text {tree }}(1,2,3,4,5)\right|^{2}+c_{2}\left|A_{5}^{\text {tree }}(1,4,3,2,5)\right|^{2}\right. \\
& \left.+c_{3}\left(A_{5}^{\text {tree }}(1,2,3,4,5) \tilde{A}_{5}^{\text {tree }}(1,4,3,2,5)+A_{5}^{\text {tree }}(1,4,3,2,5) \tilde{A}_{5}^{\text {tree }}(1,2,3,4,5)\right)\right],
\end{aligned}
$$

where $\left|A_{n}(1, \cdots, n)\right|^{2}=A_{n}(1, \cdots, n) \tilde{A}_{n}(1, \cdots, n)$. In this form the coefficients $c_{i}$ (depending only on the kinematic variables) are not always polynomials but can be rational functions of degree $n-3$.

Such relations in field theory between gravity amplitudes as sums of left/right products of color stripped gauge theories have been confirmed $[4,54,57]$ and explicitly presented for an arbitrary number of legs in [22]. These relations do not require the existence of an underlying consistent string theory and hold in any dimensions or massless matter $[58,59]$. In particular field theoretic derivations of relations of the form (3.5) have been presented in [56] and using helicity formalism in four dimensions in $[55,60]$.

\subsection{Relations between $\mathscr{N}=4$ Super Yang-Mills and $\mathscr{N}=8$ Supergravity}

The KLT relations presented in the previous section indicate that at the field theory level the relation between gravity amplitudes and Yang-Mills amplitudes amounts to replacing gauge degree of freedom of the Yang-Mills fields by Lorentz degrees of freedoms as follows

$$
A_{\mu}^{a} \rightarrow \zeta_{\mu}{ }^{a}
$$


Such a correspondence is compatible with extended supersymmetry and can be used naïvely to promote $\mathscr{N}=4$ super-Yang-Mills invariants into higher-derivative $\mathscr{N}=8$ super-invariants.

Since the full diffeomorphism invariance carries even more symmetry (For example Ricci cycling identities $R_{\mu[v \rho \sigma]}=0$ ) it is however not possible to capture all of these by such a map [61]. The naïve application of the above substitution rule on the $\partial^{2} F^{4}$ operator would for instance lead to a $\mathscr{N}=8$ supergravity amplitude with an apparent factorization of the operator $D^{2} R^{4}$. This would consequently make the $L=2$ four-graviton amplitude diverge in $D=6$ dimensions. This is however contrary to explicit knowledge as this amplitude has been shown to be finite up to $D \leq 6$ dimensions [62].

The main cause for this is that diffeomorphism invariance for $\mathscr{N}=8$ supergravity implies a summation over all permutations of external legs. Thereby the invariant $D^{2} R^{4} \sim(s+t+u) R^{4}=0$ vanish by on-shell momentum conservation (the corresponding Yang-Mills operator $\partial^{2} \operatorname{tr}\left(F^{4}\right)=$ $u \operatorname{tr}\left(F_{1} F_{2} F_{3} F_{4}\right)+t \operatorname{tr}\left(F_{1} F_{2} F_{4} F_{3}\right)+s \operatorname{tr}\left(F_{1} F_{3} F_{2} F_{4}\right)$ does not vanish on-shell because different color orderings have different kinematic factors). The first non-vanishing contribution is therefore of order $D^{4} R^{4}$. This provides a suitable structure for the two-loop amplitude kinematic factor. For the higher-loop amplitudes which have $\beta_{L} \geq 3$ for $L \geq 3[13,16]$ this does however neither give the correct form.

One thus have to be careful with such arguments since gravity theories carry symmetries beyond what is provided via two copies of the gauge transformations of Yang-Mills theories.

An important consequence of the full crossing symmetry in gravity theories is that infrared divergences in quantum gravity can be treated as in QED. They are also much milder than in color ordered theories like QCD. Hence although it looks like gravitational interactions are much more complicated than gauge theory ones they have in fact additional simplicity. This is also the case in QED which similarly has full crossing symmetry. This has the consequence that one-loop QED and $\mathscr{N}=8$ supergravity loop amplitudes enjoy extra cancelations leading to the no-triangle property $[11,26]$.

\subsection{No-triangle property of $\mathscr{N}=8$ supergravity amplitudes}

Historically computations of one-loop amplitudes for $\mathscr{N}=4$ super Yang-Mills and $\mathscr{N}=8$ supergravity was first carried out by Green, Schwarz and Brink in ref. [63]. They obtained the four point one-loop amplitude in both theories by taking the low energy limit of string theory:

$$
\begin{aligned}
& \mathscr{A}^{\text {loop }}(1,2,3,4)=K_{\text {open }} \times I_{4}(s, t), \\
& \mathscr{M}^{\text {loop }}(1,2,3,4)=K_{\text {closed }}\left(I_{4}(s, t)+I_{4}(s, u)+I_{4}(t, u)\right) .
\end{aligned}
$$

Here we have given the partial amplitude for the ordering of the external legs $(1,2,3,4)$ and set $K_{\text {open }}=s t \times A_{4}^{\text {tree }}(1,2,3,4)=t_{8}^{m_{1} \cdots m_{8}} \prod_{i=1}^{4} F_{m_{2 i-1} m_{2 i}}^{(i)}$ and defined $K_{\text {closed }}=s t u \times \mathscr{M}_{4}^{\text {tree }}(1,2,3,4)=$ $K_{\text {open }} \tilde{K}_{\text {open }}=\kappa^{4} t_{8} t_{8} \hat{R}^{4}$. In this formula $I_{4}(s, t)$ denotes the scalar box integral with legs attached in the leading in color order 1234. $s, t$ and $u$ denotes the usual Mandelstam variables (coupling constants have here been suppressed).

In the case of $\mathscr{N}=8$ supergravity the loop momentum factor in the numerator of the $n$ graviton one-loop amplitude in eq. (2.3) is given by a polynomial of degree at most $2 n-8$. Eight 
powers of momenta are canceled by supersymmetry. This implies the factorization of the operator of dimension eight in front of the loop amplitude

$$
\mathscr{P}_{2 n}(\ell) \sim \mathscr{O}_{8} \ell^{2 n-8}, \text { for } \ell \sim \infty .
$$

A power counting of infrared divergences based on the traditional color ordered Passarino-Veltman reduction (see figure 2) of $\mathscr{N}=8$ supergravity imply that the one-loop amplitude with $n \geq 7$ gravitons would contain integral functions other than scalar box integrals, i.e. scalar triangles and bubbles and rational polynomials. However explicit computations in ref. [22] of the five and sixpoint $M H V$ amplitudes evaluated using unitarity techniques surprisingly showed the amplitude to consist solely of box integral functions. It was conjectured that this behavior should hold for all $M H V$ amplitudes via consistency of factorizations and an all- $n$ ansatz consisting of box functions in the $M H V$ case was presented. In ref. [23] $\mathscr{N}=8$ supergravity were reanalyzed in the context of new results and it was postulated that the 'only boxes' property was a general feature of all $\mathscr{N}=8$ amplitudes in all helicity configurations. In ref. [24,26] this was phrased the "no-triangle hypothesis" for $\mathscr{N}=8$ and it was explicitly checked to hold for six-point $N M H V$ amplitudes and higher.

The non-presence of all contributions more singular than the scalar box amplitude has the same origin as in the case of the QED amplitude [10-12]. It is firstly due to crossing symmetry and secondly to the decoupling of longitudinal modes from the diffeomorphism gauge invariance. For theories with crossing symmetry the unordered reduction formula depicted (see figure 3) should be applied.

A generalization of this result for multi-loop amplitudes is not straightforward since no generic basis of integral functions are known for an arbitrary number of legs. The requirement of crossing symmetry in colorless theories demands the presence of both planar and non-planar integrals in the amplitude. This makes the construction of a basis of integral functions that captures the ultraviolet and infrared behavior of higher loop amplitudes even more elusive [64-67].

\subsection{Higher-loop Amplitudes}

At $L$ loop order linearized on-shell supersymmetry implies that the critical dimension for ultraviolet divergences in the four-graviton amplitude is given by

$$
D \geq 2+\frac{c_{\mathscr{N}}}{L}
$$

indicating that supergravity theories are always finite in two dimensions. In the case when $c_{\mathscr{N}}$ is bounded from above the loop order for the appearance of the first logarithmic divergence is determined by the value of $6 \leq c_{\mathscr{N}} \leq 18 . c_{\mathscr{N}}$ depends on the implementation of the linearized onshell supersymmetries and determines also the mass dimension of the first possible counter-term to the theory $[14,68-71]$.

A $L$ loop $n$-graviton amplitude has mass dimension

$$
\left[\mathscr{M}_{n ; L}^{(D)}\right]=\operatorname{mass}^{(D-2) L+2} .
$$

The low energy limit of the four-graviton amplitude at $L$ loops reads

$$
\left[\mathscr{M}_{4 ; L}^{(D)}\right]=\operatorname{mass}^{(D-2) L-\left(6+2 \beta_{L}\right)} \partial^{2 \beta_{L}} R^{4},
$$


where we have used that $\mathscr{N}=8$ supergravity four-graviton amplitudes have a factor of $R^{4}$ and allowed $2 \beta_{L}$ powers of derivatives to be distributed on the four Riemann tensors. The critical dimension for ultraviolet divergences is given by

$$
D \geq 2+\frac{6+2 \beta_{L}}{L}
$$

When $\beta_{L}=L$ at each loop order two extra powers of the external momenta are factorized and the critical dimension for ultraviolet divergences is given by $[13,14]$

$$
D \geq D_{c}=4+\frac{6}{L} \text {. }
$$

This is the same critical dimension as $\mathscr{N}=4$ super-Yang-Mills. By explicit evaluation of the amplitude it has been shown to be valid in field theory up to four-loop order [16-18].

As soon as $\beta_{L}$ is bounded after some loop order, the theory will have an ultraviolet divergence in four dimensions. The pure spinor formalism gives a counting of supersymmetric zero modes which are valid in all dimensions between $4 \leq D \leq 11$ where $\mathscr{N}=8$ supergravity can be defined. This construction implies [72] that $\beta_{L}=L$ is valid for $L \leq 6$ as long as the small $\lambda$ regulator is not needed [40]. After six-loop order one needs to use the fully regulated version of the formalism [73] and the question whether $\beta_{L}$ still can increase is open [74]. In the case when $\beta_{L}=6$ for $L \geq 6$ the critical dimension for the ultraviolet divergence is given by $D \geq 2+18 / L$ according to (3.18). This indicates that in four dimensions the first divergence would occur at nine-loop order [14].

The rule $\beta_{L}=L$ is the optimal one for finiteness in four dimensions. When the increasing the loop order $L$, with a growth of $\beta_{L}$ slower than $L$ finiteness of the theory in four dimensions is not possible. On the other hand if $\beta_{L}$ grows faster than $L$, the theory would too be finite. For instance the $L$ loop (planar and non planar) ladder diagrams of the four-graviton amplitudes are all two-particle cut constructible and given by scalar $\varphi^{3}$ diagrams with a prefactor satisfying the rule $\beta_{L}=2(L-1)$. These diagrams are ultraviolet finite for $D \leq 6$. This means that the leading ultraviolet divergences of $\mathscr{N}=8$ amplitudes are not contained in these ladder diagrams.

The absence of triangles and bubbles at one-loop order implies via general factorization theorems that higher-loop amplitudes cannot contain diagrams factorisable in one-loop amplitudes where triangles or bubbles are present. This constraint affects the structure of the higher loop amplitude [15] but is not a sufficient condition for perturbative finiteness. For this further subtle cancelations between triangle free contributions are required [16].

\section{Conclusions}

The past two years have witnessed significant progress in the computation of loop amplitudes in gauge theories. This progress for amplitude computations can be extended, in many cases, to theories incorporating gravity. Via this our understanding perturbative maximal $\mathscr{N}=8$ supergravity have improved.

It is clear that the rôle of extended supersymmetry in perturbative $\mathscr{N}=8$ supergravity is beyond the superspace transformation properties of the product of two $\mathscr{N}=4$ super-Yang-Mills theories. Actually the vacuum structure of $\mathscr{N}=4$ super-Yang-Mills and $\mathscr{N}=8$ supergravity 
theories are very different. The one of $\mathscr{N}=8$ supergravity is given in four dimensions by the homogeneous space of $E_{7(7)} /\left(S U(8)_{R} / \mathbb{Z}_{2}\right)$ [75]. While the transformational properties of the local symmetry group $S U(8)_{R}$ is like a 'square' of the group $S U(4)_{R}$ (corresponding to each $\mathscr{N}=4$ super-Yang-Mills theory) there is no corresponding symmetry of the global group $E_{7(7)}$ in $\mathscr{N}=4$ super-Yang-Mills.

The $E_{7(7)}$ symmetry rotates the different vacua of $\mathscr{N}=8$ supergravity and thus relates the perturbative contributions of the theory to the non-perturbative black hole production at high-energy. This is required for a consistent definition of the theory [76].

The search for a fundamental theory of quantum gravity is still ongoing. In these years we are gaining a much needed understanding of the concepts behind the formulation of such a theory. In supergravity theories the rôle of string theory dualities is important for their quantization and thus give us a framework for gathering further knowledge about quantum gravity, its fundamental degrees of freedom and its relation to gauge theory. Resolving the questions of ultraviolet finiteness of $\mathscr{N}=8$ supergravity and the validity of the $\beta_{L}=L$ rule [13] would indeed be remarkable and provide huge implications for non-supersymmetric low-energy descriptions of quantum gravity theories.

\section{Acknowledgements}

(NEJBB) is Knud Højgaard Assistant Professor at the Niels Bohr International Academy. The research of (NEJBB) was supported in part by grant DE-FG0290ER40542 of the US Department of Energy and (PV) by the ANR grant BLAN06-3-137168. (NEJBB) would like to thank the organizers of the workshop for financial support.

\section{References}

[1] C. Amsler et al. [Particle Data Group], "Review of particle physics," Phys. Lett. B 667 (2008) 1.

[2] S. Weinberg, "Phenomenological Lagrangians," Physica A 96, 327 (1979).

[3] J. F. Donoghue, "General Relativity As An Effective Field Theory: The Leading Quantum Corrections," Phys. Rev. D 50, 3874 (1994) [gr-qc/9405057];

N. E. J. Bjerrum-Bohr, “Leading quantum gravitational corrections to scalar QED,” Phys. Rev. D 66, 084023 (2002) [hep-th/0206236];

N. E. J. Bjerrum-Bohr, J. F. Donoghue and B. R. Holstein, "Quantum corrections to the Schwarzschild and Kerr metrics," Phys. Rev. D 68, 084005 (2003) [Erratum-ibid. D 71, 069904 (2005)] [hep-th/0211071]; "Quantum gravitational corrections to the nonrelativistic scattering potential of two masses," Phys. Rev. D 67, 084033 (2003) [Erratum-ibid. D 71, 069903 (2005)] [hep-th/0211072];

B. R. Holstein and A. Ross, "Spin Effects in Long Range Gravitational Scattering," 0802.0716 [hep-ph].

[4] Z. Bern, "Perturbative quantum gravity and its relation to gauge theory," Living Rev. Rel. 5, 5 (2002) [gr-qc/0206071].

[5] E. Witten, "Perturbative gauge theory as a string theory in twistor space," Commun. Math. Phys. 252, 189 (2004) [hep-th/0312171]. 
[6] Z. Bern, L. J. Dixon and D. A. Kosower, "On-Shell Methods in Perturbative QCD,” Annals Phys. 322 (2007) 1587 [0704.2798 [hep-ph]].

[7] N. Arkani-Hamed, F. Cachazo and J. Kaplan, "What is the Simplest Quantum Field Theory?," 0808.1446 [hep-th];

N. Arkani-Hamed, F. Cachazo, C. Cheung and J. Kaplan, "The S-Matrix in Twistor Space," arXiv:0903.2110 [hep-th]; “A Duality For The S Matrix,” arXiv:0907.5418 [hep-th].

[8] Z. Bern, J. J. M. Carrasco and H. Johansson, "Progress on Ultraviolet Finiteness of Supergravity," arXiv:0902.3765 [hep-th].

[9] J. M. Drummond, J. Henn, G. P. Korchemsky and E. Sokatchev, "Dual superconformal symmetry of scattering amplitudes in N=4 super-Yang-Mills theory," arXiv:0807.1095 [hep-th];

J. M. Drummond and J. M. Henn, “All tree-level amplitudes in N=4 SYM,” JHEP 0904, 018 (2009) [arXiv:0808.2475 [hep-th]];

J. M. Drummond, J. Henn, G. P. Korchemsky and E. Sokatchev, "Generalized unitarity for N=4 super-amplitudes," arXiv:0808.0491 [hep-th];

J. M. Drummond, M. Spradlin, A. Volovich and C. Wen, "Tree-Level Amplitudes in N=8

Supergravity," Phys. Rev. D 79, 105018 (2009) [arXiv:0901.2363 [hep-th]];

J. M. Drummond, J. M. Henn and J. Plefka, "Yangian symmetry of scattering amplitudes in N=4 super Yang-Mills theory,” JHEP 0905, 046 (2009) [arXiv:0902.2987 [hep-th]].

[10] N. E. J. Bjerrum-Bohr and P. Vanhove, "Absence of Triangles in Maximal Supergravity Amplitudes," JHEP 0810 (2008) 006 [0805.3682 [hep-th]].

[11] N. E. J. Bjerrum-Bohr and P. Vanhove, "Explicit Cancellation of Triangles in One-loop Gravity Amplitudes," JHEP 0804 (2008) 065 [0802.0868 [hep-th]].

[12] S. Badger, N. E. J. Bjerrum-Bohr and P. Vanhove, "Simplicity in the Structure of QED and Gravity Amplitudes," JHEP 0902, 038 (2009) [0811.3405 [hep-th]].

[13] M. B. Green, J. G. Russo and P. Vanhove, "Non-renormalisation conditions in type II string theory and maximal supergravity," JHEP 0702, 099 (2007) [hep-th/0610299].

[14] M. B. Green, J. G. Russo and P. Vanhove, “Ultraviolet properties of maximal supergravity,” Phys. Rev. Lett. 98, 131602 (2007) [hep-th/0611273].

[15] Z. Bern, L. J. Dixon and R. Roiban, "Is N = 8 supergravity ultraviolet finite?," Phys. Lett. B 644 (2007) 265 [hep-th/0611086].

[16] Z. Bern, J. J. Carrasco, L. J. Dixon, H. Johansson, D. A. Kosower and R. Roiban, “Three-Loop Superfiniteness of N=8 Supergravity,” Phys. Rev. Lett. 98 (2007) 161303 [hep-th/0702112].

[17] Z. Bern, J. J. M. Carrasco, L. J. Dixon, H. Johansson and R. Roiban, Phys. Rev. D 78, 105019 (2008) [0808.4112 [hep-th]].

[18] Z. Bern, J. J. Carrasco, L. J. Dixon, H. Johansson and R. Roiban, "The Ultraviolet Behavior of N=8 Supergravity at Four Loops,” Phys. Rev. Lett. 103 (2009) 081301 [arXiv:0905.2326 [hep-th]].

[19] P. S. Howe and K. S. Stelle, “The Ultraviolet Properties Of Supersymmetric Field Theories,” Int. J. Mod. Phys. A 4, 1871 (1989).

[20] P. S. Howe and K. S. Stelle, “Supersymmetry counterterms revisited,” Phys. Lett. B 554, 190 (2003) [arXiv:hep-th/0211279].

[21] G. Bossard, P. S. Howe and K. S. Stelle, "The ultraviolet question in maximally supersymmetric field theories," Gen. Rel. Grav. 41 (2009) 919 [arXiv:0901.4661 [hep-th]]. 
[22] Z. Bern, L. J. Dixon, M. Perelstein and J. S. Rozowsky, "Multi-leg one-loop gravity amplitudes from gauge theory,” Nucl. Phys. B 546 (1999) 423 [arXiv:hep-th/9811140].

[23] Z. Bern, N. E. J. Bjerrum-Bohr and D. C. Dunbar, "Inherited twistor-space structure of gravity loop amplitudes," JHEP 0505, 056 (2005) [hep-th/0501137].

[24] N. E. J. Bjerrum-Bohr, D. C. Dunbar and H. Ita, "Six-point one-loop N = 8 supergravity NMHV amplitudes and their IR behaviour,” Phys. Lett. B 621, 183 (2005) [hep-th/0503102].

[25] N. E. J. Bjerrum-Bohr, D. C. Dunbar, H. Ita, W. B. Perkins and K. Risager, "MHV-vertices for gravity amplitudes," JHEP 0601, 009 (2006) [hep-th/0509016].

[26] N. E. J. Bjerrum-Bohr, D. C. Dunbar, H. Ita, W. B. Perkins and K. Risager, "The no-triangle hypothesis for N = 8 supergravity," JHEP 0612 (2006) 072 [hep-th/0610043].

[27] Z. Bern, J. J. Carrasco, D. Forde, H. Ita and H. Johansson, "Unexpected Cancellations in Gravity Theories," Phys. Rev. D 77 (2008) 025010 [0707.1035 [hep-th]].

[28] N. E. J. Bjerrum-Bohr and P. Vanhove, "On Cancellations of Ultraviolet Divergences in Supergravity Amplitudes,” Fortsch. Phys. 56, 824 (2008) [arXiv:0806.1726 [hep-th]].

[29] S. Mandelstam, "Light Cone Superspace And The Ultraviolet Finiteness Of The N=4 Model," Nucl. Phys. B 213, 149 (1983).

[30] L. Brink, O. Lindgren and B. E. W. Nilsson, "The Ultraviolet Finiteness Of The N=4 Yang-Mills Theory," Phys. Lett. B 123 (1983) 323.

[31] N. Marcus and A. Sagnotti, "The Ultraviolet Behavior Of N=4 Yang-Mills And The Power Counting Of Extended Superspace,” Nucl. Phys. B 256, 77 (1985).

[32] E. A. Bergshoeff and M. de Roo, "The Quartic Effective Action Of The Heterotic String And Supersymmetry,” Nucl. Phys. B 328, 439 (1989).

[33] M. de Roo, H. Suelmann and A. Wiedemann, "The Supersymmetric effective action of the heterotic string in ten-dimensions,” Nucl. Phys. B 405, 326 (1993) [arXiv:hep-th/9210099].

[34] A. A. Tseytlin, “On SO(32) heterotic - type I superstring duality in ten dimensions,” Phys. Lett. B 367, 84 (1996) [arXiv:hep-th/9510173].

[35] A. A. Tseytlin, "Heterotic - type I superstring duality and low-energy effective actions," Nucl. Phys. B 467, 383 (1996) [arXiv:hep-th/9512081].

[36] C. Bachas, C. Fabre, E. Kiritsis, N. A. Obers and P. Vanhove, "Heterotic/type-I duality and D-brane instantons," Nucl. Phys. B 509, 33 (1998) [arXiv:hep-th/9707126].

[37] Z. Bern, L. J. Dixon, D. C. Dunbar, M. Perelstein and J. S. Rozowsky, “On the relationship between Yang-Mills theory and gravity and its implication for ultraviolet divergences,” Nucl. Phys. B 530, 401 (1998) [arXiv:hep-th/9802162].

[38] Z. Bern, J. J. M. Carrasco, H. Johansson and D. A. Kosower, "Maximally supersymmetric planar Yang-Mills amplitudes at five loops,” Phys. Rev. D 76, 125020 (2007) [arXiv:0705.1864 [hep-th]].

[39] M. B. Green, J. H. Schwarz and E. Witten, "Superstring Theory," Cambridge, Uk: Univ. Pr. ( 1987) ( Cambridge Monographs On Mathematical Physics).

[40] N. Berkovits, M. B. Green, J. G. Russo and P. Vanhove, "Non-renormalization conditions for four-gluon scattering in supersymmetric string and field theory," JHEP 0911 (2009) 063 [arXiv:0908.1923 [hep-th]]. 
[41] J. M. Drummond, J. Henn, V. A. Smirnov and E. Sokatchev, "Magic identities for conformal four-point integrals,” JHEP 0701, 064 (2007) [arXiv:hep-th/0607160].

[42] Z. Bern, L. J. Dixon, D. C. Dunbar and D. A. Kosower, "One loop n point gauge theory amplitudes, unitarity and collinear limits,” Nucl. Phys. B 425, 217 (1994) [hep-ph/9403226].

[43] Z. Bern, L. J. Dixon, D. C. Dunbar and D. A. Kosower, "Fusing gauge theory tree amplitudes into loop amplitudes,” Nucl. Phys. B 435, 59 (1995) [hep-ph/9409265].

[44] L. M. Brown and R. P. Feynman, "Radiative corrections to Compton scattering," Phys. Rev. 85 (1952) 231;

G. Passarino and M. J. G. Veltman, "One Loop Corrections For $e^{+} e^{-}$Annihilation Into $\mu^{+} \mu^{-}$In The Weinberg Model,” Nucl. Phys. B 160 (1979) 151.

[45] J. M. Campbell, E. W. N. Glover and D. J. Miller, "One-loop tensor integrals in dimensional regularisation,” Nucl. Phys. B 498 (1997) 397 [hep-ph/9612413].

[46] A. Denner and S. Dittmaier, "Reduction schemes for one-loop tensor integrals," Nucl. Phys. B 734 (2006) 62 [hep-ph/0509141].

[47] G. Passarino and M. J. G. Veltman, "One Loop Corrections For $e^{+} e^{-}$Annihilation Into $\mu^{+} \mu^{-}$In The Weinberg Model,” Nucl. Phys. B 160 (1979) 151.

[48] S. Weinberg, “Infrared photons and gravitons," Phys. Rev. 140 (1965) B516.

[49] Z. Bern, L. J. Dixon and D. A. Kosower, "All next-to-maximally helicity-violating one-loop gluon amplitudes in N = 4 super-Yang-Mills theory,” Phys. Rev. D 72 (2005) 045014 [arXiv:hep-th/0412210].

[50] G. Mahlon, “One loop multi - photon helicity amplitudes,” Phys. Rev. D 49, 2197 (1994) [arXiv:hep-ph/9311213].

[51] A. Brandhuber, P. Heslop and G. Travaglini, "One-Loop Amplitudes in N=4 Super Yang-Mills and Anomalous Dual Conformal Symmetry,” arXiv:0905.4377 [hep-th].

[52] B.S. De Witt, "Quantum Theory of Gravity. I. The Canonical Theory”, Phys. Rev., Vol. 160, No. 5, 1113, 1967; “ Quantum Theory of Gravity. II. The Manifestly Covariant Theory”, Phys. Rev., Vol. 162, No. 5, 1195, 1967; " Quantum Theory of Gravity. III. Applications of the Covariant Theory”, Phys. Rev., Vol. 162, No. 5, 1239, 1967.

[53] S. Sannan, “Gravity As The Limit Of The Type Ii Superstring Theory,” Phys. Rev. D 34, 1749 (1986).

[54] H. Kawai, D. C. Lewellen and S. H. H. Tye, "A Relation Between Tree Amplitudes Of Closed And Open Strings,” Nucl. Phys. B 269, 1 (1986).

[55] H. Elvang and D. Z. Freedman, "Note on graviton MHV amplitudes,” JHEP 0805 (2008) 096 [arXiv:0710.1270 [hep-th]];

M. Bianchi, H. Elvang and D. Z. Freedman, "Generating Tree Amplitudes in N=4 SYM and N = 8 SG,” JHEP 0809, 063 (2008) [arXiv:0805.0757 [hep-th]];

H. Elvang, D. Z. Freedman and M. Kiermaier, "Recursion Relations, Generating Functions, and Unitarity Sums in N=4 SYM Theory,” JHEP 0904, 009 (2009) [arXiv:0808.1720 [hep-th]];

H. Elvang, D. Z. Freedman and M. Kiermaier, "Proof of the MHV vertex expansion for all tree amplitudes in N=4 SYM theory,” JHEP 0906, 068 (2009) [arXiv:0811.3624 [hep-th]].

[56] Z. Bern, J. J. M. Carrasco and H. Johansson, “New Relations for Gauge-Theory Amplitudes,” Phys. Rev. D 78 (2008) 085011 [arXiv:0805.3993 [hep-ph]]. 
[57] F. A. Berends, W. T. Giele and H. Kuijf, "On Relations Between Multi - Gluon And Multigraviton Scattering," Phys. Lett. B 211, 91 (1988).

[58] N. E. J. Bjerrum-Bohr, "String theory and the mapping of gravity into gauge theory," Phys. Lett. B 560, 98 (2003) [hep-th/0302131]; “Generalized string theory mapping relations between gravity and gauge theory,” Nucl. Phys. B 673, 41 (2003) [hep-th/0305062];

N. E. J. Bjerrum-Bohr and K. Risager, "String theory and the KLT-relations between gravity and gauge theory including external matter,” Phys. Rev. D 70, 086011 (2004) [hep-th/0407085].

[59] Z. Bern, A. De Freitas and H. L. Wong, “On the coupling of gravitons to matter," Phys. Rev. Lett. 84, 3531 (2000) [hep-th/9912033].

[60] J. M. Drummond, M. Spradlin, A. Volovich and C. Wen, "Tree-Level Amplitudes in N=8 Supergravity," arXiv:0901.2363 [hep-th].

[61] K. Peeters, P. Vanhove and A. Westerberg, "Supersymmetric higher-derivative actions in ten and eleven dimensions, the associated superalgebras and their formulation in superspace," Class. Quant. Grav. 18 (2001) 843 [hep-th/0010167].

[62] Z. Bern, L. J. Dixon, D. C. Dunbar, M. Perelstein and J. S. Rozowsky, "On the relationship between Yang-Mills theory and gravity and its implication for ultraviolet divergences," Nucl. Phys. B 530, 401 (1998) [hep-th/9802162].

[63] M. B. Green, J. H. Schwarz and L. Brink, "N=4 Yang-Mills And N=8 Supergravity As Limits Of String Theories," Nucl. Phys. B 198 (1982) 474.

[64] Z. Bern, L. J. Dixon, D. A. Kosower, R. Roiban, M. Spradlin, C. Vergu and A. Volovich, "The Two-Loop Six-Gluon MHV Amplitude in Maximally Supersymmetric Yang-Mills Theory,” Phys. Rev. D 78 (2008) 045007 [0803.1465 [hep-th]].

[65] F. Cachazo, "Sharpening The Leading Singularity," 0803.1988 [hep-th].

[66] F. Cachazo, M. Spradlin and A. Volovich, "Leading Singularities of the Two-Loop Six-Particle MHV Amplitude,” Phys. Rev. D 78, 105022 (2008) [0805.4832 [hep-th]].

[67] M. Spradlin, A. Volovich and C. Wen, “Three-Loop Leading Singularities and BDS Ansatz for Five Particles,” Phys. Rev. D 78, 085025 (2008) [0808.1054 [hep-th]].

[68] P. S. Howe and U. Lindstrom, "Higher Order Invariants In Extended Supergravity,” Nucl. Phys. B 181 (1981) 487.

[69] R. E. Kallosh, “Counterterms in extended supergravities,” Phys. Lett. B 99 (1981) 122.

[70] P. S. Howe, K. S. Stelle and P. K. Townsend, "Miraculous Ultraviolet Cancellations In Supersymmetry Made Manifest,” Nucl. Phys. B 236 (1984) 125.

[71] P. S. Howe and K. S. Stelle, "Supersymmetry counterterms revisited," Phys. Lett. B 554 (2003) 190 [hep-th/0211279].

[72] N. Berkovits, “New higher-derivative R**4 theorems,” Phys. Rev. Lett. 98 (2007) 211601 [hep-th/0609006].

[73] N. Berkovits and N. Nekrasov, "Multiloop superstring amplitudes from non-minimal pure spinor formalism,” JHEP 0612, 029 (2006) [arXiv:hep-th/0609012].

[74] P. A. Grassi and P. Vanhove, "Higher-loop amplitudes in the non-minimal pure spinor formalism," JHEP 0905 (2009) 089 [arXiv:0903.3903 [hep-th]]. 
[75] E. Cremmer and B. Julia, “The N=8 Supergravity Theory. 1. The Lagrangian,” Phys. Lett. B 80 (1978) 48. “The SO(8) Supergravity,” Nucl. Phys. B 159 (1979) 141.

[76] M. B. Green, H. Ooguri and J. H. Schwarz, "Nondecoupling of maximal supergravity from the superstring," Phys. Rev. Lett. 99, 041601 (2007). 